\title{
Optimal Collision Avoidance and Formation Switching on Riemannian Manifolds ${ }^{1}$
}

\author{
Jianghai Hu and Shankar Sastry \\ \{jianghai,sastry\}@eecs.berkeley.edu \\ Department of Electrical Eng. \& Comp. Science \\ University of California at Berkeley
}

\begin{abstract}
In this paper the problems of optimal collision avoidance and optimal formation switching for multiple agents moving on a Riemannian manifold are studied. It is assumed that the underlying manifold admits a group of isometries, with respect to which the Lagrangian function is invariant. Reduction method is used to derive optimality conditions for the solutions. Some examples are presented.
\end{abstract}

\section{Motivation}

We study two related problems for multiple agents moving on a Riemannian manifold: the optimal collision avoidance (OCA) and the optimal formation switching (OFS). In each case, the trajectories of the agents must satisfy the separation constraint that at any time the riemannian distance between any two of them is at least $r$ for some positive $r$. In the OFS problem, the distances between certain pairs of agents are further required to be $r$. The optimal (joint) trajectories are the ones that minimize the weighted sum of the trajectory energies of individual agents, with the weights representing the priorities of the agents.

The motivating application for this research is aircraft conflict resolution $[1,2]$, in which the underlying manifold is $\mathbb{R}^{2}$ or $\mathbb{R}^{3}$, and $r$ is 5 nautical miles for en route aircraft. Related applications can be, for example, multiple mobile robots cooperating to carry a common object, or a multi-link reconfigurable robot performing configuration switching. In this paper we consider only holonomic constraints, as opposed to the many treatments dealing with nonholonomic constraints, such as $[3,4,5]$. Other relevant papers include $[6,7]$.

In many applications, it is often the case that the underlying manifold admits a group of symmetries. In the case of a smooth state space, the classical Noether

\footnotetext{
${ }^{1}$ Research supported by DARPA under grant F33615-98-C3614 and NSF KDI 9873474. The first author would like to thank Professor Alan Weinstein for his helpful discussions and comments.
}

theorem $[8,9]$ can be used to reduce the dimension of the problem by establishing the conservation of certain quantities called the momentum maps. The problems we consider here have nonsmooth boundary constraints. However, since the constraints are also invariant with respect to the symmetries, the conservation laws still apply. In particular, if the number of agents is small, these laws may help to characterize the solutions.

This paper is organized as following. The OCA and OFS problems are formulated in Section 2. In section 3, we focus on the case when the underlying manifold admits a group of symmetries, and establish the conservation of momentum maps. To illustrate the results, a simple example of three agents moving on the plane is presented in Section 4. We show that the conservation laws enable us to characterize the solutions in a certain sense. Finally Section 5 contains some concluding remarks.

\section{Problem Formulation}

In this section the problems we are going to study are formulated in their most generality.

Let $M$ be a $C^{\infty}$ Riemannian manifold. Given $t_{0}, t_{1} \in \mathbb{R}$ with $t_{0} \leq t_{1}$, the arc length of a curve ${ }^{1} \gamma:\left[t_{0}, t_{1}\right] \rightarrow$ $M$ is defined as $\int_{t_{0}}^{t_{1}}\|\dot{\gamma}(t)\| d t$, where $\|\cdot\|$ denotes the norm of tangent vectors associated with the riemannian metric $\langle\cdot, \cdot\rangle$. The distance between two arbitrary points $a$ and $b$ in $M, d_{M}(a, b)$, is by definition the infimum of the arc lengths of all the curves connecting $a$ and $b$. A geodesic in $M$ is a locally distance-minimizing curve. In this paper, we always assume that $M$ is connected and complete, and that all the geodesics are parameterized proportionally to arc length.

Let $L: T M \rightarrow \mathbb{R}$ be a smooth function defined on the tangent bundle of $M$ (the Lagrangian function). As an example one can take $L=\frac{1}{2}\|\cdot\|^{2}$. For each curve

\footnotetext{
${ }^{1}$ All curves in this paper are assumed to be continuous and piecewise $C^{\infty}$, unless otherwise stated
} 
$\gamma:\left[t_{0}, t_{1}\right] \rightarrow M$, its cost is defined as

$$
J(\gamma)=\int_{t_{0}}^{t_{1}} L[\dot{\gamma}(t)] d t .
$$

The curve joining two fixed points in $M$ with minimal cost is an instance of the extremals of the functional $J$, which in any local coordinates of $T M$, $\left(q_{1}, \ldots, q_{m}, \dot{q}_{1}, \ldots, \dot{q}_{m}\right), m=\operatorname{dim}(M)$, can be characterized by the Euler-Lagrange equations [8]:

$$
\frac{d}{d t} \frac{\partial L}{\partial \dot{q}_{i}}=\frac{\partial L}{\partial q_{i}}, \quad i=1, \ldots, m
$$

By choosing $L=\frac{1}{2}\|\cdot\|^{2}$, the above equations describe the geodesics in $M([10])$.

Consider an (ordered) $k$-tuple of points of $M,\left\langle q_{i}\right\rangle_{i=1}^{k}=$ $\left(q_{1}, \ldots, q_{k}\right)$. We say that $\left\langle q_{i}\right\rangle_{i=1}^{k}$ satisfies the $r$ separation condition for some positive $r$ if and only if $d_{M}\left(q_{i}, q_{j}\right) \geq r$ for all $i \neq j$. Let $\left\langle a_{i}\right\rangle_{i=1}^{k}$ and $\left\langle b_{i}\right\rangle_{i=1}^{k}$ be two $k$-tuples of points of $M$, each of which satisfies the $r$-separation condition. $\left\langle a_{i}\right\rangle_{i=1}^{k}$ is called the starting position and $\left\langle b_{i}\right\rangle_{i=1}^{k}$ the destination position.

Let $h=\left(h_{1}, \ldots, h_{k}\right)$ be a $k$-tuple of curves in $M$ defined on $\left[t_{0}, t_{1}\right]$ such that $h_{i}\left(t_{0}\right)=a_{i}, h_{i}\left(t_{1}\right)=b_{i}$, for $i=1, \ldots, k$. One can think of $h$ as the joint trajectory of $k$ agents moving on $M$ which starts from $a_{i}$ at time $t_{0}$ and ends at $b_{i}$ at time $t_{1}$ for $i=1, \ldots, k$ respectively. $h$ is said to be collision-free if the $k$-tuple $\left\langle h_{i}(t)\right\rangle_{i=1}^{k}$ satisfies the $r$-separation condition for each $t \in\left[t_{0}, t_{1}\right]$. Alternatively, if each agent is a disk of radius $\frac{r}{2}$ in $M$, then $h$ is collision-free if and only if the corresponding joint trajectory satisfies that no two agents overlap throughout the encounter $\left[t_{0}, t_{1}\right]$. Naturally, $r$ is chosen to be small enough so that it is possible to pack $k$ disks of radius $\frac{r}{2}$ in $M$.

The first problem we are going to study is

Problem 1 (OCA) Among all collision-free $h=$ $\left\langle h_{i}\right\rangle_{i=1}^{k}$ which start from $\left\langle a_{i}\right\rangle_{i=1}^{k}$ and end at $\left\langle b_{i}\right\rangle_{i=1}^{k}$, find the one (or ones) minimizing the cost

$$
J(h)=\sum_{i=1}^{k} \lambda_{i} J\left(h_{i}\right) .
$$

Here $\left\langle\lambda_{i}\right\rangle_{i=1}^{k}$ is a $k$-tuple of positive numbers representing the relative priorities of the $k$ agents, while $J\left(h_{i}\right)$ is defined in (1) for each $i$.

There is an alternative way of formulating the OCA problem. By viewing each $k$-tuple of points of $M$ as a single point in $M^{(k)}=M \times \ldots \times M, h$ becomes a curve in $M^{(k)}$ starting from $a=\left(a_{1}, \ldots, a_{k}\right)$ at time $t_{0}$ and ending at $b=\left(b_{1}, \ldots, b_{k}\right)$ at time $t_{1}$, while avoiding the obstacle $W$ defined by

$$
W=\cup_{i \neq j}\left\{\left(x_{1}, \ldots, x_{k}\right) \in M^{(k)}: d_{M}\left(x_{i}, x_{j}\right)<r\right\} .
$$

Hence solutions to Problem 1 are cost-minimizing curves in $M^{(k)} \backslash W$ between $a$ and $b$. If $L=\frac{1}{2}\|\cdot\|^{2}$ and all $\lambda_{i}$ 's are identical, solutions are geodesics in $M^{(k)} \backslash W$, a manifold with nonsmooth boundary.

To introduce the second problem we need some notions. Let $\left\langle q_{i}\right\rangle_{i=1}^{k}$ be a $k$-tuple of points of $M$ satisfying the $r$-separation condition. Then a graph $(V, E)$ called the formation pattern of $\left\langle q_{i}\right\rangle_{i=1}^{k}$ can be constructed as following: the set of vertices is $V=\{1, \ldots, k\}$, and the set of edges $E$ is such that an edge $e_{i j}$ between vertex $i$ and vertex $j$ exists if and only if $d_{M}\left(q_{i}, q_{j}\right)=r$. For a collision-free $k$-tuple of curves $h=\left\langle h_{i}\right\rangle_{i=1}^{k}$ in $M$ defined on $\left[t_{0}, t_{1}\right]$, the formation pattern of $h$ at time $t \in\left[t_{0}, t_{1}\right]$ is defined to be the formation pattern of $\left\langle h_{i}(t)\right\rangle_{i=1}^{k}$.

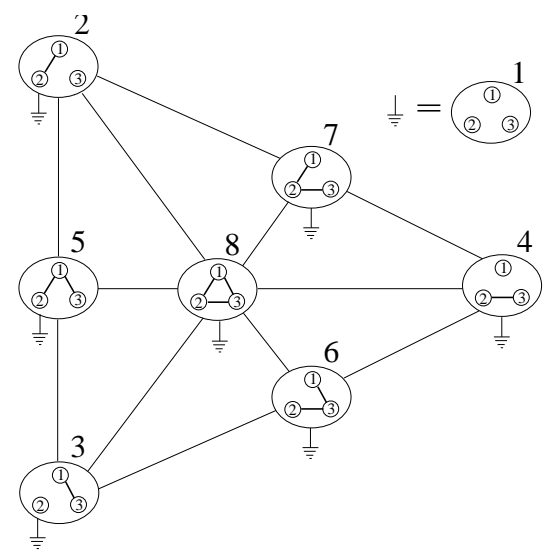

Figure 1: Formation adjacency graph.

Depending on $M, r$, and $k$, not all graphs with $k$ vertices can be realized as the formation pattern of some $\left\langle q_{i}\right\rangle_{i=1}^{k}$. Two possible formation patterns are called adjacent if either of them is a subgraph of the other. This relation can be used to define a graph $G_{a d j}$ called the formation adjacency graph, whose set of vertices is the set of all possible formation patterns, and whose set of edges is such that an edge connects two formation patterns if and only if they are adjacent. Figure 1 shows $G_{a d j}$ in the case $M=\mathbb{R}$ and $k=3$, where the attachments of the "ground" symbol to vertices $2, \ldots, 8$ signal their adjacency with vertex 1 .

Now we are ready to define the second problem.

Problem 2 (OFS) Let $G_{a d j}^{\prime}$ be a connected subgraph of $G_{a d j}$ such that the formation patterns of $\left\langle a_{i}\right\rangle_{i=1}^{k}$ and $\left\langle b_{i}\right\rangle_{i=1}^{k}$ are both vertices of $G_{a d j}^{\prime}$. Among all collisionfree $h$ which start from $\left\langle a_{i}\right\rangle_{i=1}^{k}$, end in $\left\langle b_{i}\right\rangle_{i=1}^{k}$, and satisfy the additional constraint that the formation pattern of $h$ at any time $t \in\left[t_{0}, t_{1}\right]$ belongs to the vertices of $G_{a d j}^{\prime}$, find the one (or ones) minimizing the cost (2).

In the example shown in Figure 1 , one can choose $G_{a d j}^{\prime}$ to be the subgraph consisting of vertices $5,6,7,8$, and all the edges among them, thus requiring that all three 
agents, each of which is of radius $\frac{r}{2}$, have to "contact" each other either directly or indirectly at all time. Alternatively, $G_{a d j}^{\prime}$ can be the subgraph consisting of vertices $2,5,7,8$, and all the edges among them. So agent 1 and 2 have to be bound together at all time, and the OFS problem becomes the optimal collision avoidance between agent 3 and this two-agent subsystem.

For certain $G_{a d j}^{\prime}$, the OFS problem may not have a solution. For example in Figure 1, if $G_{a d j}^{\prime}$ consists of only vertex 1 , then the corresponding subset of $M^{(k)} \backslash W=\mathbb{R}^{6} \backslash W$ is its interior. For any two points $a, b \in \operatorname{int}\left(\mathbb{R}^{6} \backslash W\right)$ that are not 'visible' to each other with respect to the obstacle $W$ (i.e., the line segment connecting them is not fully contained in $\operatorname{int}\left(\mathbb{R}^{6} \backslash W\right)$ ), the OFS problem does not admit a solution. Therefore some constraints on $G_{a d j}^{\prime}$ have to be imposed to ensure that a solution exists for the OFS problem. This is guaranteed if $G_{a d j}^{\prime}$ has the following property: for each formation pattern $(V, E)$ belonging to the vertices of $G_{a d j}^{\prime}$, any formation pattern $\left(V^{\prime}, E^{\prime}\right)$ containing $(V, E)$ as a subgraph is also a vertex of $G_{a d j}^{\prime}$. We call such a $G_{a d j}^{\prime}$ closed, since the subset of $M^{(k)} \backslash W$ it corresponds to is closed. In the following, we will always assume $G_{a d j}^{\prime}$ to be closed.

\section{Symmetry Reduction}

Instead of working on general Riemannian manifolds, we focus on the special case when $M$ admits a group of symmetries. More precisely, we assume:

1. $\Phi: G \times M \rightarrow M$ is a $C^{\infty}$ left action of a Lie group $G$ on $M$ by isometries.

2. The Lagrangian function $L$ is $G$-invariant.

For brevity, we write $g x=\Phi(g, x)$. For each $g \in G$, define $\Phi_{g}: M \rightarrow M$ to be the map $x \mapsto g x, \forall x \in M$. Similarly, for each $x \in M$ define $\Phi^{x}: G \rightarrow M$ to be the map $g \mapsto g x, \forall g \in G$. Both $\Phi_{g}$ and $\Phi^{x}$ are $C^{\infty}$ maps. That $\Phi$ is a left action is equivalent to that $\Phi_{e}=\mathrm{id}_{M}$, where $e$ is the identity of $G$, and that for $g_{1}, g_{2} \in G$, $\Phi_{g_{1} g_{2}}=\Phi_{g_{1}} \circ \Phi_{g_{2}}$. For each $g \in G$, assumption 1 implies that $\Phi_{g}$ is an isometry of $M$, while assumption 2 implies that $L \circ d \Phi_{g}=L$. Here $d \Phi_{g}: T M \rightarrow T M$ is the tangent map of $\Phi_{g}$.

Examples of such $M$ and $G$ include

Example 1 Let $M=\mathbb{R}^{n}, L=\frac{1}{2}\|\cdot\|^{2}$. Let $G=\mathbf{S E}_{n}$ be the group of rigid body motions of $\mathbb{R}^{n}$.

Example 2 Let $M=\mathbf{S}^{n-1}$ be the $(n-1)$-sphere in $\mathbb{R}^{n}$ with the standard metric. Let $G=\mathbf{S O}_{n}$ be the group of rotations. Let $L=\frac{1}{2}\|\cdot\|^{2}$.
Example 3 Let $M=G$ be a Lie group equipped with a left invariant riemannian metric. Then the group multiplication $G \times G \rightarrow G$ is a left action of $G$ on itself by isometries. Let $L: T G \rightarrow \mathbb{R}$ be any left invariant function. Such L's correspond in a one-to-one way with functions $l: \mathfrak{g}=T_{e} G \rightarrow \mathbb{R}$.

In mechanics there is the well-known Noether theorem, which roughly states that for every symmetry there corresponds an integral of motion [8, 9]. This is also the case here. In general, there are two equivalent ways of deriving the conserved quantities, the Lagrangian and the Hamiltonian, which are related by the Legendre transform [8]. In this paper we adopt the more direct Lagrangian point of view for two reasons. First the nonsmooth nature of the problems can be more easily dealt with this way; second some modification of the arguments can lead to global results which are otherwise hard to obtain. These will be explored in future work.

Suppose $h=\left\langle h_{i}\right\rangle_{i=1}^{k}$ is an optimal solution to the OCA (or OFS) problem. We shall derive necessary conditions on $h$ in the following way. Let $g:(-\epsilon, \epsilon) \times\left[t_{0}, t_{1}\right] \rightarrow G$ be a $C^{\infty}$ proper variation of the constant identity map $c_{e}$ mapping every $t \in\left[t_{0}, t_{1}\right]$ to $e \in G$, i.e. $g\left(\cdot, t_{0}\right)=$ $g\left(\cdot, t_{1}\right)=g(0, \cdot) \equiv e$, where $\epsilon$ is a small positive number. Then for each $s \in(-\epsilon, \epsilon), g_{s}(\cdot)=g(s, \cdot)$ is a $C^{\infty}$ curve in $G$ both starting and ending at $e$, hence can be used to define a $k$-tuple of curves $h_{s}=\left\langle h_{s, i}\right\rangle_{i=1}^{k} \triangleq\left\langle g_{s} h_{i}\right\rangle_{i=1}^{k}$, which starts from $\left\langle a_{i}\right\rangle_{i=1}^{k}$ and ends in $\left\langle b_{i}\right\rangle_{i=1}^{k}$. Moreover, $h_{s}$ is collision-free and has the same formation pattern at all time as $h$ by assumption 1 . Notice that $h_{0}=h$. Define $J(s)=J\left(h_{s}\right)$. Then a necessary condition for $h$ to be optimal is that $\frac{d J}{d s}(0)=0$.

For each $(s, t) \in(-\epsilon, \epsilon) \times\left[t_{0}, t_{1}\right]$, denote

$$
\dot{g}_{s}(t)=\dot{g}(s, t)=\frac{\partial g}{\partial t}(s, t), g_{s}^{\prime}(t)=g^{\prime}(s, t)=\frac{\partial g}{\partial s}(s, t),
$$

both of which belong to $T_{g(s, t)} G$. Define

$$
\begin{aligned}
& \xi_{s}(t)=\xi(s, t)=g(s, t)^{-1} \dot{g}(s, t), \\
& \eta_{s}(t)=\eta(s, t)=g(s, t)^{-1} g^{\prime}(s, t) .
\end{aligned}
$$

Here to simplify notation we use $g(s, t)^{-1} \dot{g}(s, t)$ to denote $d T_{g(s, t)^{-1}}[\dot{g}(s, t)]$ (for any $g \in G, T_{g}: G \rightarrow G$ stands for the left multiplication by $g$, while $d T_{g}$ is its tangent map). Similarly for $g(s, t)^{-1} g^{\prime}(s, t)$. This kind of notational simplifications will be carried out in the following without further explanation. Both $\xi(s, t)$ and $\eta(s, t)$ belong to $T_{e} G=\mathfrak{g}$, the Lie algebra of $G$. The fact that $g$ is a proper variation implies that $g^{\prime}\left(\cdot, t_{0}\right)=g^{\prime}\left(\cdot, t_{1}\right)=0$, hence $\eta\left(\cdot, t_{0}\right)=\eta\left(\cdot, t_{1}\right)=0$. Moreover, $g(0, \cdot)=e$ implies that $\dot{g}(0, \cdot)=0$, hence $\xi_{0}(\cdot)=0$.

Lemma 1 ([11]) Let $\xi^{\prime}(s, t)=\frac{\partial \xi}{\partial s}(s, t)$ and $\dot{\eta}(s, t)=$ $\frac{\partial \eta}{\partial t}(s, t)$. Then $\xi^{\prime}=\dot{\eta}+[\xi, \eta]$. 
Define $\omega(t)=\xi_{0}^{\prime}(t), \forall t \in\left[t_{0}, t_{1}\right]$. Then by letting $s=0$ in Lemma 1, we have $\omega=\dot{\eta}_{0}+\left[\xi_{0}, \eta_{0}\right]=\dot{\eta}_{0}$ since $\xi_{0} \equiv$ 0 . So $\int_{t_{0}}^{t_{1}} \omega d t=\eta_{0}\left(t_{1}\right)-\eta_{0}\left(t_{0}\right)=0$. Conversely, for each $\omega:\left[t_{0}, t_{1}\right] \rightarrow \mathfrak{g}$ with $\int_{t_{0}}^{t_{1}} \omega d t=0$, we can define $g(s, t)=\exp _{e}\left[s \int_{t_{0}}^{t} \omega(t) d t\right], \forall(s, t) \in(-\epsilon, \epsilon) \times\left[t_{0}, t_{1}\right]$ for some $\epsilon>0$ small enough, which is a proper variation of $c_{e}$ with $\omega=\xi_{0}^{\prime}$, where $\xi=g^{-1} \dot{g}$. Therefore,

Lemma $2 A C^{\infty}$ map $\omega:\left[t_{0}, t_{1}\right] \rightarrow \mathfrak{g}$ can be realized as $\omega=\xi_{0}^{\prime}$ where $\xi=g^{-1} \dot{g}$ for some $C^{\infty}$ proper variation $g$ of $c_{e}$ if and only if $\int_{t_{0}}^{t_{1}} \omega d t=0$.

Suppose one such $g$ is chosen. For each $(s, t) \in(-\epsilon, \epsilon) \times$ $\left[t_{0}, t_{1}\right], i=1, \ldots, k$, we have ${ }^{2}$

$$
L\left[\dot{h}_{s, i}\right]=L\left[\dot{g}_{s} h_{i}+g_{s} \dot{h}_{i}\right]=L\left[\xi_{s} h_{i}+\dot{h}_{s}\right],
$$

by the $G$-invariance of $L$. Here $\dot{g}_{s} h_{i}=d \Phi^{h_{i}}\left(\dot{g}_{s}\right)$, and $g_{s} \dot{h}_{i}=d \Phi_{g_{s}}\left(\dot{h}_{i}\right)$. The cost of $h_{s}$ is

$$
J(s)=\sum_{i=1}^{k} \lambda_{i} \int_{t_{0}}^{t_{1}} L\left[\xi_{s} h_{i}+\dot{h}_{i}\right] d t .
$$

For any vector space $V$, denote with $(\cdot, \cdot): V^{*} \times V \rightarrow \mathbb{R}$ the natural pairing between $V$ and its dual $V^{*}$. Differentiating (4) at $s=0$, one gets

$$
\begin{aligned}
J^{\prime}(0) & =\left.\sum_{i=1}^{k} \lambda_{i} \int_{t_{0}}^{t_{1}}\left(d L_{\xi_{s} h_{i}+\dot{h}_{i}}, \xi_{s}^{\prime} h_{i}\right) d t\right|_{s=0} \\
& =\sum_{i=1}^{k} \lambda_{i} \int_{t_{0}}^{t_{1}}\left(d L_{\dot{h}_{i}}, d \Phi^{h_{i}}(\omega)\right) d t \\
& =\int_{t_{0}}^{t_{1}}\left(\sum_{i=1}^{k} \lambda_{i}\left(d \Phi^{h_{i}}\right)^{*} d L_{\dot{h}_{i}}, \omega\right) d t
\end{aligned}
$$

where $d L_{\dot{h}_{i}} \in T_{h_{i}}^{*} M$ is in fact the differential at $\dot{h}_{i}$ of $\left.L\right|_{T_{h_{i}} M}$, the restriction of $L$ on $T_{h_{i}} M .\left(d \Phi^{h_{i}}\right)^{*}$ : $T_{h_{i}}^{*} M \rightarrow \mathfrak{g}^{*}$ is the dual of $d \Phi^{h_{i}}: \mathfrak{g} \rightarrow T_{h_{i}} M$, i.e.,

$$
\left(\left(d \Phi^{h_{i}}\right)^{*} u, v\right)=\left(u, d \Phi^{h_{i}} v\right), \forall u \in T_{h_{i}}^{*} M, v \in \mathfrak{g} .
$$

From (5) and Lemma 2, the condition that $J^{\prime}(0)=0$ for all proper variation $g$ of $c_{e}$ is equivalent to

$$
\int_{t_{0}}^{t_{1}}\left(\sum_{i=1}^{k} \lambda_{i}\left(d \Phi^{h_{i}}\right)^{*} d L_{\dot{h}_{i}}, \omega\right) d t=0,
$$

for all $\omega:\left[t_{0}, t_{1}\right] \rightarrow \mathfrak{g}$ such that $\int_{t_{0}}^{t_{1}} \omega d t=0$. Note that $\sum_{i=1}^{k} \lambda_{i}\left(d \Phi^{h_{i}}\right)^{*} d L_{\dot{h}_{i}}$ is piecewise $C^{\infty}$, so we have

\footnotetext{
${ }^{2}$ Since $h_{i}$ is only piecewise $C^{\infty}$, this and all equations that follow should be understand to hold only for those $t$ where $\dot{h}_{i}$ 's are well defined.
}

Theorem 1 (Noether) Suppose $h=\left\langle h_{i}\right\rangle_{i=1}^{k}$ is an optimal solution to the OCA (or OFS) problem. Then there exists a constant $\nu_{0} \in \mathfrak{g}^{*}$ such that

$$
\nu \triangleq \sum_{i=1}^{k} \lambda_{i}\left(d \Phi^{h_{i}}\right)^{*} d L_{\dot{h}_{i}} \equiv \nu_{0}
$$

for all $t \in\left[t_{0}, t_{1}\right]$ where $\dot{h}_{i}$ 's are well defined.

If the boundary constraints are ignored, then $\nu$ defined in (7) as a function of $\dot{h}_{i}$ 's is in fact the momentum map for the left action of $G$ on $M^{(k)}$ with Lagrangian function $\sum_{i=1}^{k} \lambda_{i} L\left(\dot{h}_{i}\right)([9])$. The point of Theorem 1 is that this momentum map is still conserved in the presence of obstacles, even when the state space is obtained by piecing together cells in a nonsmooth way.

If $L=\frac{1}{2}\|\cdot\|^{2}$, the conclusion of Theorem 1 can be simplified by canonically identifying each $u \in T_{h_{i}} M$ with the element in $T_{h_{i}}^{*} M$ defined by $v \mapsto\langle u, v\rangle, \forall v \in$ $T_{h_{i}} M$. Thus $d L_{\dot{h}_{i}}$ is identified with $\dot{h}_{i}$, and (7) becomes

$$
\sum_{i=1}^{k} \lambda_{i}\left(d \Phi^{h_{i}}\right)^{*} \dot{h}_{i} \equiv \nu_{0} \in \mathfrak{g}^{*}
$$

where $\left(d \Phi^{h_{i}}\right)^{*}: T_{h_{i}} M \rightarrow \mathfrak{g}$ is now defined by

$$
\left(\left(d \Phi^{h_{i}}\right)^{*} u, v\right)=\left\langle u, d \Phi^{h_{i}} v\right\rangle, \forall u \in T_{h_{i}} M, v \in \mathfrak{g} .
$$

In Example 1, it can be shown that the conserved quantities are the classical linear momentum and the (generalized) angular momentum of a $k$-particle system moving on $\mathbb{R}^{n}$. In Example 2, (7) becomes the conservation of angular momentum of a $k$-particle system moving on $\mathbf{S}^{n-1}$. In both cases, the $k$ particles have masses $\lambda_{1}, \ldots, \lambda_{k}$. We illustrate this only for Example 2. For any $u \in T_{h_{i}} \mathbf{S}^{n-1}$ and any $v \in \mathfrak{s o}_{n}$,

$$
\left\langle u, d \Phi^{h_{i}} v\right\rangle=\left\langle u, v h_{i}\right\rangle=u^{t} v h_{i}=\left\langle u h_{i}^{t}, v\right\rangle_{F},
$$

where $\langle\cdot, \cdot\rangle_{F}$ is the Frobenius inner product on $\mathbb{R}^{n \times n}$ defined by $\langle A, B\rangle=\operatorname{tr}\left(A^{t} B\right\rangle, \forall A, B \in \mathbb{R}^{n \times n}$. Since $v$ is skew-symmetric, we have

$$
\left\langle u h_{i}^{t}, v\right\rangle_{F}=\frac{1}{2}\left\langle u h_{i}^{t}-h_{i} u^{t}, v\right\rangle_{F},
$$

where $u h_{i}^{t}-h_{i} u^{t}$ is skew-symmetric, hence belongs to $\mathfrak{s o}_{n}$. Combining the above two equations with (9), we have $\left(\left(d \Phi^{h_{i}}\right)^{*} u, v\right)=\frac{1}{2}\left\langle u h_{i}^{t}-h_{i} u^{t}, v\right\rangle_{F}, \forall v \in \mathfrak{s o}_{n}$. So $\left(d \Phi^{h_{i}}\right)^{*} u=u h_{i}^{t}-h_{i} u^{t}$ under the identification of $\mathfrak{s o}_{n}$ with $\mathfrak{s o}_{n}^{*}$ via $\frac{1}{2}\langle\cdot, \cdot\rangle_{F}$, and (8) becomes

$$
\sum_{i=1}^{k} \lambda_{i}\left(\dot{h}_{i} h_{i}^{t}-h_{i} \dot{h}_{i}^{t}\right) \equiv \nu_{0} \in \mathfrak{s o}_{n},
$$

In coordinates, if $h_{i}=\left(h_{i, 1}, \ldots, h_{i, n}\right) \in \mathbf{S}^{n-1}$, then $\sum_{i=1}^{k} \lambda_{i}\left(\dot{h}_{i j_{1}} h_{i j_{2}}^{t}-h_{i j_{1}} \dot{h}_{i j_{2}}^{t}\right) \equiv C_{j_{1} j_{2}}$ for all $j_{1} \neq j_{2}$. In 
particular, if $n=3,(10)$ can be written compactly as $\sum_{i=1}^{k} \lambda_{i}\left(h_{i} \times \dot{h}_{i}\right) \equiv \Omega_{0}$ for some $\Omega_{0} \in \mathbb{R}^{3}$, where $\times$ is the vector product. So the total angular momentum is conserved.

Next we study a special case of Example 3 for which the conclusion of Theorem 1 takes an especially simple form. Let $M=G$ be a Lie group with a bi-invariant riemannian metric (i.e., a metric that is invariant under both left and right multiplications. Let the action $\Phi$ : $G \times G \rightarrow G$ be the left multiplication. Choose $L=$ $\frac{1}{2}\|\cdot\|^{2}$. Suppose $h=\left\langle h_{i}\right\rangle_{i=1}^{k}$ is a solution to the OCA (or OFS) problem. Then $\forall u \in T_{h_{i}} M, v \in \mathfrak{g}$,

$$
\left\langle u, d \Phi^{h_{i}} v\right\rangle=\left\langle u, v h_{i}\right\rangle=\left\langle u h_{i}^{-1} h_{i}, v h_{i}\right\rangle=\left\langle u h_{i}^{-1}, v\right\rangle .
$$

Therefore, we have

$$
\left(\left(d \Phi^{h_{i}}\right)^{*} u, v\right)=\left\langle u h_{i}^{-1}, v\right\rangle, \quad \forall v \in \mathfrak{g} .
$$

Under the canonical identification of $\mathfrak{g}$ with $\mathfrak{g}^{*}$ via $\langle\cdot, \cdot\rangle$, the last equation is equivalent to

$$
\left(d \Phi^{h_{i}}\right)^{*} u=u h_{i}^{-1} \in \mathfrak{g}, \quad \forall u \in T_{h_{i}} M .
$$

Therefore the conservation law (8) can now be simplified to

$$
\sum_{i=1}^{k} \lambda_{i} \dot{h}_{i} h_{i}^{-1} \equiv \nu_{0} \in \mathfrak{g} .
$$

In the simplest case, if $M$ is a Euclidean space $\mathbb{R}^{n}$ or a flat $n$-torus $\mathbf{T}^{n}$ with the canonical metric and with addition as the group operation, then (12) implies the conservation of linear momentum.

\section{An Example}

In this section, we apply the results in the previous section to the OFS problem when $M=\mathbb{R}^{2}$ and $G=\mathbf{S E}_{2}$. We assume $k=3, r=1$, so the formation adjacency graph $G_{a d j}$ is plotted in Figure 1. Let $G_{a d j}^{\prime}$ be the subgraph of $G_{a d j}$ consisting of vertices $5,6,7,8$ and all the edges among them. Therefore, in steering the three agents from their starting position $\left\langle a_{i}\right\rangle_{i=1}^{3}$ to their destination position $\left\langle b_{i}\right\rangle_{i=1}^{3}$, it is required that at any time $t \in\left[t_{0}, t_{1}\right]$, there exists (at least) one agent whose distances with the rest two agents are both 1 .

Suppose $L=\frac{1}{2}\|\cdot\|^{2}$. Then the cost defined in (2) coincides with the sum of the integrals of kinetic energy of the three agents with masses $\lambda_{1}, \lambda_{2}, \lambda_{3}$ respectively. Therefore by the Hamiltonian principle of least action [8], for each formation pattern, a solution to the OFS problem restricted to this particular formation pattern corresponds to the motion of a multi-link system moving on the plane with no external forces acting on it. The multi-link system consists of three particles (agents) of masses $\lambda_{1}, \lambda_{2}, \lambda_{3}$ respectively, and there is a rod of zero mass and length 1 between particle $i$ and particle $j$ if and only if $e_{i j}$ is an edge of this formation pattern. If $\left\langle a_{i}\right\rangle_{i=1}^{3}$ and $\left\langle b_{i}\right\rangle_{i=1}^{3}$ belong to different formation patterns, then a switching between formation patterns must occur at some $t \in\left[t_{0}, t_{1}\right]$. During the switching, the velocities of the three agents will in general experience discontinuous changes.

However, by applying Theorem 1, we conclude that for any solution $\left\langle h_{i}\right\rangle_{i=1}^{3}$ to the OFS problem, the total linear momentum $\sum_{i=1}^{3} \lambda_{i} \dot{h}_{i}$ and the total angular momentum $\sum_{i=1}^{3} \lambda_{i} h_{i}^{t} R_{\pi / 2} \dot{h}_{i}$ are both constant throughout $\left[t_{0}, t_{1}\right]$. Here $R_{\pi / 2}$ is the 2 by 2 matrix of rotation by $\frac{\pi}{2}$ counterclockwise. This is true even when there are switchings between different formation patterns. Moreover, since in this case solutions as curves in $M^{(k)}$ are geodesics in a certain manifold with boundary, the total energy $\sum_{i=1}^{3} \lambda_{i}\left\|\dot{h}_{i}\right\|^{2}$ is also conserved.

It turns out that these conserved quantities are enough to characterize the solutions. For simplicity we assume that $\lambda_{1}=\lambda_{2}=\lambda_{3}$.

Proposition 1 ([12]) If $\left\langle h_{i}\right\rangle_{i=1}^{3}$ is a solution to the OFS problem with starting position $\left\langle a_{i}\right\rangle_{i=1}^{3}$ and destination position $\left\langle b_{i}\right\rangle_{i=1}^{3}$. Then for any $w \in \mathbb{R}^{2}$, a solution to the OFS problem with starting position $\left\langle a_{i}\right\rangle_{i=1}^{3}$ and destination position $\left\langle b_{i}+w\right\rangle_{i=1}^{3}$ is $\left\langle\hat{h}_{i}\right\rangle_{i=1}^{3}$, where $\hat{h}_{i}(t)=h_{i}(t)+\frac{t-t_{0}}{t_{1}-t_{0}} w, \forall t \in\left[t_{0}, t_{1}\right]$.

An intuitive way to understand Proposition 1 is that the solutions to the OFS problem correspond to the motions of a physical system, hence are invariant with respect to the choice of inertial coordinate systems, say, the one moving at constant speed $-w$ relative to the stationary one.

By choosing an appropriate $w$ if necessary, we can ensure that $\frac{1}{3} \sum_{i=1}^{3} a_{i}=\frac{1}{3} \sum_{i=1}^{3} b_{i}$. Proposition 1 implies that we only need to solve the OFS problem for this special case. In particular, we shall in the following assume that $\frac{1}{3} \sum_{i=1}^{3} a_{i}=\frac{1}{3} \sum_{i=1}^{3} b_{i}=0$. Since the linear momentum is conserved, the solution $\left\langle h_{i}\right\rangle_{i=1}^{3}$ in this case must satisfy $\sum_{i=1}^{3} h_{i}(t) \equiv 0$, i.e., the three-agent system has its center of masses stationary at 0 . As a result, $\left\langle h_{i}\right\rangle_{i=1}^{3}$ as a curve in $\mathbb{R}^{6}$ is always contained in the four dimensional subspace $V$ of $\mathbb{R}^{6}$ defined by $\left\{\left(x_{1}, y_{1}, x_{2}, y_{2}, x_{3}, y_{3}\right): \sum_{i=1}^{3} x_{i}=\sum_{i=1}^{3} y_{i}=0\right\}$. This reduces the dimension of the problem by 2 .

Each vertex in $G_{a d j}^{\prime}$ is a formation pattern, which corresponds to a smooth submanifold of $V \backslash W$. For example, vertex 5 corresponds to $\left\{\left(x_{1}, y_{1}, x_{2}, y_{2}, x_{3}, y_{3}\right) \in\right.$ $\left.V: d_{12}=d_{13}=1, d_{23}>1\right\}$, where $d_{i j}=$ $\sqrt{\left(x_{i}-x_{j}\right)^{2}+\left(y_{i}-y_{j}\right)^{2}}, 1 \leq i, j \leq 3$. Denote this set with $X_{5}$. If we define $\theta_{12}$ (respectively, $\theta_{13}$ ) to be the angle $\left(x_{2}-x_{1}, y_{2}-y_{1}\right)$ (respectively, $\left.\left(x_{3}-x_{1}, y_{3}-y_{1}\right)\right)$ 


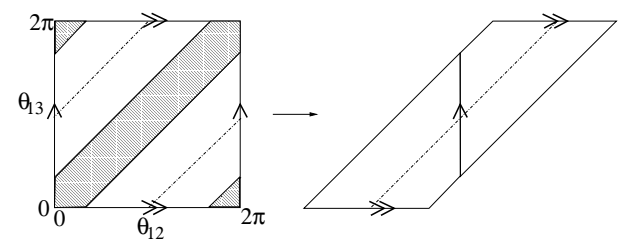

Figure 2: $\hat{X}_{5}$

makes with the positive $x$-axis of $\mathbb{R}^{2}$, then $\left(\theta_{12}, \theta_{13}\right)$ is a global coordinate of $X_{6}$, taking values in the rectangle $[0,2 \pi] \times[0,2 \pi]$ with the opposite edges identified, i.e. the 2-torus $\mathbf{T}^{2}$. Moreover, the shaded region in Figure 2 has to be removed from $\mathbf{T}^{2}$ in order to satisfy the constraint $d_{23}>1$, resulting in a subset $\hat{X}_{5}$ homeomorphic to $S^{1} \times(0,1)$, an untwisted ribbon whose boundary consists of two disjoint circles. Similarly, vertex 6 and 7 correspond to sets $X_{6}$ and $X_{7}$ respectively, both of which are also untwisted ribbons. Moreover, $X_{5}, X_{6}$ and $X_{7}$ share the same boundary, which is exactly the set vertex 8 corresponds to, namely, $X_{8}=$ $\left\{\left(x_{1}, y_{1}, x_{2}, y_{2}, x_{3}, y_{3}\right) \in V: d_{12}=d_{13}=d_{23}=1\right\} . X_{8}$ consists of two connected components since in forming the equilateral triangle, agent 1, 2, and 3 can be counted either clockwise or counterclockwise.

A solution $\left\langle h_{i}\right\rangle_{i=1}^{3}$ to the OFS problem restricted to the domain of any particular formation pattern is a geodesic in that domain. After some computation, geodesics in $X_{5}$ are governed by [12]

$$
\begin{aligned}
& \ddot{\theta}_{12}=\frac{-\sin \left[2\left(\theta_{12}-\theta_{13}\right)\right]\left(\dot{\theta}_{12}\right)^{2}+4 \sin \left(\theta_{12}-\theta_{13}\right)\left(\dot{\theta}_{13}\right)^{2}}{2\left[4-\cos ^{2}\left(\theta_{12}-\theta_{13}\right)\right]} \\
& \ddot{\theta}_{13}=\frac{-4 \sin \left(\theta_{12}-\theta_{13}\right)\left(\dot{\theta}_{12}\right)^{2}+\sin \left[2\left(\theta_{12}-\theta_{13}\right)\right]\left(\dot{\theta}_{13}\right)^{2}}{2\left[4-\cos ^{2}\left(\theta_{12}-\theta_{13}\right)\right]}
\end{aligned}
$$

Total angular momentum of the three agents in the $\left(\theta_{12}, \theta_{13}\right)$ coordinate is

$$
K_{1}\left(\dot{\theta}_{12}+\dot{\theta}_{13}\right)\left[2-\cos \left(\theta_{12}-\theta_{13}\right)\right],
$$

for some constant $K_{1}$. Total kinetic energy is

$$
K_{2}\left[\left(\dot{\theta}_{12}\right)^{2}+\left(\dot{\theta}_{13}\right)^{2}-\cos \left(\theta_{12}-\theta_{13}\right) \dot{\theta}_{12} \dot{\theta}_{13}\right],
$$

for some constant $K_{2}$. At the boundary of $X_{5}, \theta_{12}-$ $\theta_{13}= \pm \frac{\pi}{3}$, so (14) and (15) become $\frac{3}{2} K_{1}\left(\dot{\theta}_{12}+\dot{\theta}_{13}\right)$ and $\frac{1}{2} K_{2}\left[2\left(\dot{\theta}_{12}\right)^{2}+2\left(\dot{\theta}_{13}\right)^{2}-\dot{\theta}_{12} \dot{\theta}_{13}\right]$ respectively. Similar results hold for geodesics in $X_{6}$ and $X_{7}$.

Consider now the switching from formation pattern 5 to 6 via formation pattern 8 at some point in $X_{8}$. Let $\left(\theta_{31}, \theta_{32}\right)$ be the coordinate of $X_{6}$. Then the above analysis implies that

$$
\begin{aligned}
\dot{\theta}_{12}+\dot{\theta}_{13} & =\dot{\theta}_{31}+\dot{\theta}_{32} \\
2\left(\dot{\theta}_{12}\right)^{2}+2\left(\dot{\theta}_{13}\right)^{2}-\dot{\theta}_{12} \dot{\theta}_{13} & =2\left(\dot{\theta}_{31}\right)^{2}+2\left(\dot{\theta}_{32}\right)^{2}-\dot{\theta}_{31} \dot{\theta}_{32}
\end{aligned}
$$

These two equations will determine uniquely $\dot{\theta}_{31}$ and $\dot{\theta}_{32}$ after the switching subject to the constraint that they must point inside of $X_{6}$.

Equations such as (13) and (16) characterize the evolution of the solutions for the OFS problem completely. Alternatively, they define the dynamics and the reset maps of a hybrid system.

\section{Conclusions}

We study the problems of optimal collision avoidance and optimal formation switching for multiple agents on a Riemannian manifold with a group of symmetries. Some necessary conditions are given, and the results are illustrated by examples.

\section{References}

[1] J. Hu, M. Prandini, and S. Sastry, "Optimal maneuver for multiple aircraft conflict resolution: a braid point of view," in Proc. 39th IEEE Int. Conf. on Dec. and Contr., 2000.

[2] J. Kuchar and L.C. Yang, "Survey of conflict detection and resolution modeling methods," in Proc. AIAA Guidance, Navigation, and Control Conf., New Orleans, LA, 1997.

[3] A. Bicchi and L. Pallottino, "Optimal planning for coordinated vehicles with bounded curvature," in Proc. Work. Algorithmic Foundation of Robotics (WAFR'2000), Dartmouth, 2000.

[4] J.P. Desai and V. Kumar, "Nonholonomic motion planning for multiple mobile manipulators," in Proceedings of IEEE Int. Conf. on Robotics and Automation, Albuquerque, NM, 1991.

[5] P. Tabuada, G.J. Pappas, and P. Lima, "Feasible formations of multi-agent systems," preprint.

[6] P. S. Krishnaprasad, "Eulerian many-body problems," in Dynamics and control of multibody systems (Brunswick, ME, 1988), pp. 187-208. Amer. Math. Soc., Providence, RI, 1989.

[7] G.C. Walsh, R. Montgomery, and S. Sastry, "Optimal path planning on matrix lie groups," in Proc. 33 Int. Conf. Dec. and Contr., 1994.

[8] V.I. Arnold, K. Vogtmann, and A. Weinstein, Mathematical Methods of Classical Mechanics, 2nd Edition, Springer-Verlag, 1989.

[9] J.E. Marsden and T.S. Ratiu, An Introduction to Mechanics and Symmetry, Springer-Verlag, 1994.

[10] M.P. de Carmo, Riemannian Geometry, Birkhäuser Boston, 1992.

[11] A.M. Bloch, P.S. Krishnaprasad, J.E. Marsden, and T.S. Ratiu, "The Euler-Poincare equations and double bracket dissipation," Comm. Math. Phys., vol. 175, no. 1, pp. 1-42, 1996.

[12] J. Hu, M. Prandini, and S. Sastry, "Hybrid geodesics as optimal solutions to the collision-free motion planning problem," in Hybrid Systems: Comp. and Contr., 4th International Workshop, 2001. 